\title{
Comparação entre a drenagem linfática manual e a massoterapia convencional para a melhora da qualidade de vida das mães da comunidade de São Luiz Gonzaga
}

As mulheres mães acumulam funções e sentem o peso dessa responsabilidade. 0 dia-a-dia com os filhos, com a família, seus interesses pessoais, as atividades profissionais, ou seja, um somatório de atribuições realizadas, na maioria das situações, de maneira isolada, sem compartilhar, o que contribui para sua rotina sobrecarregada (SPINDOLA e SANTOS, 2011). Por esses motivos, é valido às mães priorizarem uma hora do seu dia para um momento só, um momento delas, uma hora de relaxamento, em consequência, a qualidade de vida (QV) irá melhorar. O presente estudo teve como objetivo verificar qual a técnica manual mais eficaz, entre a massoterapia convencional (MC) e a drenagem linfática manual (DLM) para a melhora da qualidade de vida das mães da comunidade de São Luiz Gonzaga. Trata-se de um ensaio clínico randomizado, no qual a amostra foi composta por 20 mães, entre 22 e 55 anos, divididas em dois grupos: 10 receberam a MC e 10 receberam a DLM, submetidas a 4 sessões uma vez por semana. Como forma de avaliação da QV foi aplicado o questionário WHOQOL-bref. Os resultados do estudo mostraram que houve melhora na QV das mães pesquisadas com a intervenção das duas técnicas, porém, a técnica que obteve diferença estatisticamente significante foi a MC $(p=0,02)$, concluindo que a técnica manual mais eficaz para a melhora da QV das mães estudadas, foi à MC.

\section{Comparison between manual lymphatic drainage and conventional massage therapy to improve the quality of life of mothers in the community of São Luiz Gonzaga}

Women mothers accumulate roles and feel the burden of that responsibility. The day-to-day with the children, with the family, their personal interests, the professional activities, that is, a sum of attributions realized, in most situations, in an isolated way, without sharing, which contributes to their overloaded routine (SPINDOLA e SANTOS, 2011). For these reasons, it is up to mothers to prioritize one hour of their day for one moment, one moment for them, one hour for relaxation, as a result, quality of life (QV) will improve. The present study had as objective to verify which is the most effective manual technique, between conventional massotherapy (MC) and manual lymphatic drainage (DLM) to improve the quality of life of the mothers of the community of São Luiz Gonzaga. This was a randomized clinical trial in which the sample consisted of 20 mothers, aged 22 to 55 years, divided into two groups: 10 received MC and 10 received DLM, submitted to 4 sessions once a week. The WHOQOL-bref questionnaire was used to assess QV. The results of the study showed that there was an improvement in the QV of the mothers studied with the intervention of the two techniques, however, the technique that obtained a statistically significant difference was MC $(p=0.02)$, concluding that the most effective manual technique for improving QoL of the studied mothers was MC.

Keywords: Conventional Massage Therapy; Manual lymphatic drainage; Quality of life.

\section{Topic: Fisioterapia}

Reviewed anonymously in the process of blind peer.
Received: 10/02/2021

Approved: 20/03/2021
Tânia Regina Warpechowski (ib

Universidade Regional Integrada do Alto Uruguai e das Missões, Brasil http://lattes.cnpq.br/5042138395515098 https://orcid.org/0000-0003-0163-3339 twrfisio@yahoo.com.br
Referencing this:

WARPECHOWSKI, T. R. Comparação entre a drenagem linfática manual e a massoterapia convencional para a melhora da qualidade de vida das mães da comunidade de São Luiz Gonzaga. Scire Salutis, v.11, n.2, p.35-41, 2021. DOI: http://doi.org/10.6008/CBPC22369600.2021.002.0004 
Comparação entre a drenagem linfática manual e a massoterapia convencional para a melhora da qualidade de vida das mães da comunidade de

\section{INTRODUÇÃO}

A Organização Mundial de Saúde (OMS) define qualidade de vida (QV) como "a percepção do indivíduo de sua posição na vida, no contexto da cultura e sistema de valores nos quais ele vive e em relação aos seus objetivos, expectativas, padrões e preocupações" (MIURA, 2012). Não existe uma definição única de QV, entretanto, todas as definições revelam a ideia de que a felicidade não consiste apenas da satisfação de necessidades materiais e sim, da existência de outros aspectos de vida que não estão sendo atendidos (RADUNZ et al., 2005).

As mulheres mães acumulam funções e sentem o peso dessa responsabilidade. $\mathrm{O}$ dia-a-dia com os filhos, com a família, seus interesses pessoais, as atividades profissionais, ou seja, um somatório de atribuições realizadas, na maioria das situações, de maneira isolada, sem compartilhar, o que contribui para sua rotina sobrecarregada (SPINDOLA et al., 2011). Por esses motivos, é valido às mães priorizarem uma hora do seu dia para um momento só, um momento delas, uma hora de relaxamento, em consequência, a qualidade de vida geral (QVG) irá melhorar.

Segundo o Conselho Regional de Fisioterapia e Terapia Ocupacional da Terceira Região (CREFITO-3) a fisioterapia é uma ciência tão antiga quanto o homem, em razão de que nossos ancestrais tentavam minimizar a dor esfregando o local dolorido, o que evoluiu para as técnicas que hoje conhecemos como Exercícios Terapêuticos Manuais (HERNANDES et al., 2013). O fisioterapeuta dentro da área da Dermatofuncional dispõe de diversas técnicas manuais, dentre elas a drenagem linfática manual (DLM) e a massoterapia convencional (MC) (CARREIRO et al., 2012).

A MC tem como principal efeito o relaxamento, pois é capaz de produzir estimulação mecânica nos tecidos por aplicação rítmica de pressão e estiramento (COSTA et al., 2006). A DLM apresenta efeito relaxante e analgésico, o que ajuda na cefaleia, dor nos membros inferiores, além de melhorar a qualidade do sono (FERREIRA et al., 2010).

Sendo assim, este estudo justifica-se pela importância da temática apresentada, sendo que a qualidade de vida é importante para o ser humano, pois contribui para o bem estar físico e mental, através disso, o objetivo do estudo é verificar qual a técnica manual mais eficaz, entre a MC e a DLM para a melhora da qualidade de vida das mães da comunidade de São Luiz Gonzaga.

\section{METODOLOGIA}

Estudo caracterizado como ensaio clínico randomizado, a amostra foi composta por 20 mães, entre 22 e 55 anos, divididas em dois grupos, no qual 10 receberam a MC e 10 receberam a DLM. As intervenções foram realizadas na Clinica escola do curso de Fisioterapia da URI - São Luiz Gonzaga, tendo o total de 4 sessões uma vez por semana. 0 tempo preconizado para cada sessão foi de 45 minutos à uma hora. As intervenções foram realizadas no período dos meses de Setembro à Outubro de 2017 e, foram aplicadas pela pesquisadora e por três acadêmicas do curso de Fisioterapia da instituição. Todas as executoras da pesquisa foram treinadas pela pesquisadora responsável quanto ás técnicas de intervenção. 
Comparação entre a drenagem linfática manual e a massoterapia convencional para a melhora da qualidade de vida das mães da comunidade de

Como critérios de inclusão foram mães da comunidade de São Luiz Gonzaga; maiores de 18 anos; que relatem problemas na qualidade do sono, no ambiente de trabalho; que não tenham prazer nos momentos de lazer, nas atividades de vida diária; que relatem que não possuem tempo para momentos de relaxamento e que tiverem disponibilidade de participar da pesquisa. Já como critérios de exclusão foram mulheres não mães; menores de 18 anos; que tenham momentos de relaxamento em alguma clínica de fisioterapia e aquelas que apresentarem fraturas ósseas, doenças da pele, tumores benignos e malignos, processos infecciosos, distúrbios circulatórios e doenças degenerativas.

Este projeto foi aprovado pelo Comitê de Ética e Pesquisa da URI Campus Santiago sob o CAAE 72908017.8.0000.5353. Após a aprovação, entramos em contato com algumas mães no protocolo da URI SLG que esperavam a saída dos seus filhos das salas de aula. A partir de uma conversa informal, essas mães nos passaram os contatos de outras mães que possuem filhos que estudam em outras escolas. Todas essas mães tiveram a oportunidade de participar da pesquisa. Após este evento, todas essas mães foram solicitadas a comparecerem na clínica escola da URI - São Luiz Gonzaga com data e hora marcada, para explicar os objetivos e os procedimentos, de forma clara e simples e, em seguida, foi entregue as voluntárias o Termo de Consentimento Livre e Esclarecido (TCLE) que foi assinado pelas mesmas. Os horários e os dias das intervenções foram combinados de acordo com a disponibilidade das mães, da pesquisadora e da clínica escola.

As avaliações foram realizadas através de uma ficha de coleta dos dados, contendo os dados pessoais das voluntárias para caracterização da amostra e, para avaliar a qualidade de vida foi aplicado o instrumento WHOQOL-bref, que é composto por 26 questões, sendo duas a respeito da QV em geral, e as demais representam cada uma das 24 perguntas que compõem o instrumento original e estão divididas em quatro domínios, sendo eles, capacidade física, o bem-estar psicológico, o meio ambiente e as relações sociais (SALES et al., 2011).

As voluntárias foram conduzidas para a maca e, se posicionaram em decúbito ventral. Logo após, foram utilizadas as seguintes técnicas de MC: deslizamento superficial e profundo, amassamento, fricção e percussão. As manobras foram aplicadas nos membros inferiores, superiores e em toda a coluna vertebral, com a utilização de cremes antialérgicos manipulados. Já na aplicação da DLM, as voluntárias ficaram em decúbito dorsal na maca. Foi utilizada a técnica de Vodder, com as manobras de bombeamento, mobilizações, rotatórias, círculos estacionários e também procedimentos de captação e evacuação. Esses movimentos foram realizados em todas as regiões do corpo, exceto a face, utilizando cremes antialérgicos manipulados quando necessário.

A análise estatística foi desenvolvida no software PAST e no Microsoft Excel 2010. As variáveis qualitativas foram descritas em média, desvio padrão (DV) e porcentagem respectivamente. Foi verificada a normalidade da amostra, através do teste de Shapiro-Wilk, e ambos os grupos apresentaram distribuição normal, ou seja, p> 0,05. Para comparação entre pré e pós-intervenção dos grupos foi utilizado o Teste T pareado. Na comparação entre os grupos foi utilizado o Teste $T$ two samples, assumindo valor de $p<0,05$, como estatisticamente significante. 
Comparação entre a drenagem linfática manual e a massoterapia convencional para a melhora da qualidade de vida das mães da comunidade de

\section{RESULTADOS}

Ambos os grupos não apresentaram diferença significativa em relação à idade e IMC. Em relação à idade do grupo da $M C(37,7 \pm 8,88$, Média $\underline{\underline{D}} \mathrm{DV})$, e da $\operatorname{DLM}(37,1 \pm 8,06$, Média $\underline{\underline{ }} \mathrm{DV})$ com $p=0,8$; já em relação ao IMC (índice de massa corporal) do grupo da $M C(27,3 \pm 3,29$, Média \pm DV) e do grupo da DLM $(25,5 \pm 8,88$, Média \pm DV) com $p=0,8 ; 50 \%$ das mães eram casadas no grupo da MC e $60 \%$ no grupo da DLM; $50 \%$ tinham dois filhos no grupo da MC e $60 \%$ apenas um filho no grupo da DLM; na MC $30 \%$ eram professoras, já na DLM 40\% eram professoras, apresentados na tabela 1.

Tabela 1: Características gerais das mães submetidas à massoterapia convencional e a drenagem linfática manual.

\begin{tabular}{|c|c|c|c|}
\hline Características & $\mathrm{MC}(\mathrm{n}=10)$ & DLM $(n=10)$ & $p$ \\
\hline Idade $^{A}$ & $37,7 \pm 8,88$ & $37,1 \pm 8,06$ & 0,8 \\
\hline $\mathrm{IMC}^{\mathrm{A}}$ & $27,3 \pm 4,73$ & $25,5 \pm 3,29$ & 0,8 \\
\hline \multicolumn{4}{|l|}{ Estado Civil $^{\mathrm{B}}$} \\
\hline Solteira & $30 \%$ & $40 \%$ & \\
\hline Casada & $50 \%$ & $60 \%$ & \\
\hline Divorciada & $10 \%$ & & \\
\hline União Estável & $10 \%$ & & \\
\hline \multicolumn{4}{|l|}{$N^{0}$ de filhos ${ }^{B}$} \\
\hline $1 / 2 / 3$ & $1(40 \%) / 2(50 \%) / 3(10 \%)$ & $1(60 \%) / 2(40 \%)$ & \\
\hline \multicolumn{4}{|l|}{ Profissão $^{B}$} \\
\hline Professora & $30 \%$ & $40 \%$ & \\
\hline Fisioterapeuta & $20 \%$ & $10 \%$ & \\
\hline Estudante & $20 \%$ & $10 \%$ & \\
\hline Do lar & $20 \%$ & & \\
\hline Funcionária Pública & $10 \%$ & & \\
\hline Téc. Enfermagem & & $10 \%$ & \\
\hline Bibliotecária & & $10 \%$ & \\
\hline Administradora & & $10 \%$ & \\
\hline Farmacêutica & & $10 \%$ & \\
\hline
\end{tabular}

A - média e desvio padrão; B - porcentagem; MC: massoterapia convencional; DLM: drenagem linfática manual; IMC: índice de massa corporal; no: número; téc: técnica; $n$ : número da amostra; \%: porcentagem; $p$ : nível de significância $(p>0,05)$.

Em relação aos domínios verificados pré e pós-intervenção do grupo da $M C$, nota-se que houve diferença significativa nos domínios físico $(p=0,004)$ e meio ambiente $(p=0,009)$, indicando que houve uma melhora na percepção destes domínios. Já em relação à pré e pós-intervenção do grupo da DLM, observou-se que em todos os domínios não houve diferença significativa ( $p>0,05)$.

Ao compararmos a QVG pré e pós-intervenção, observou-se que houve diferença significativa apenas no grupo da $M C(p=0,009)$, indicando que a $M C$ foi eficiente na melhora da qualidade de vida das mães pesquisadas, conforme a tabela 2.

Ao comparar os domínios dos dois grupos na pós-intervenção, nota-se que houve diferença significativa apenas no domínio físico $(p=0,01)$. Em comparação à QVG pós-intervenção, entre os grupos da MC e da DLM, verificamos que houve diferença significativa $(p=0,02)$, apresentados na tabela 3 , ficando evidente estatisticamente que houve melhora significativa na qualidade de vida das mães pesquisadas com a intervenção das duas técnicas, porém, a técnica mais eficaz foi a MC, conforme a tabela 2. 
Comparação entre a drenagem linfática manual e a massoterapia convencional para a melhora da qualidade de vida das mães da comunidade de São Luiz Gonzaga

Tabela 2: Comparação do efeito pré e pós-intervenção entre a massoterapia convencional e a drenagem linfática manual na percepção dos domínios e da qualidade de vida em geral.

\begin{tabular}{cccc|ccc}
\hline & \multicolumn{3}{c}{ MC $(\mathrm{n}=10)$} & \multicolumn{3}{c}{ DLM $(\mathrm{n}=10)$} \\
\hline Domínios & Pré & Pós & $\mathrm{p}$ & Pré & Pós & $\mathrm{p}$ \\
\cline { 2 - 7 } Físico & $56,7 \pm 5,94$ & $63,5 \pm 6,48$ & $0,004^{*}$ & $56,4 \pm 7,29$ & $55,3 \pm 7,55$ & 0,4 \\
Psicológico & $68,7 \pm 8,60$ & $71,6 \pm 7,80$ & 0,1 & $67,5 \pm 9,16$ & $67,4 \pm 9,16$ & 0,9 \\
Social & $69,1 \pm 15,7$ & $80,8 \pm 18,0$ & 0,1 & $73,3 \pm 12,2$ & $70,8 \pm 8,10$ & 0,5 \\
Meio Ambiente & $67,8 \pm 5,32$ & $72,5 \pm 4,62$ & $0,009^{*}$ & $68,1 \pm 6,21$ & $68,1 \pm 7,49$ & 0,9 \\
QVG & $65,6 \pm 6,69$ & $72,1 \pm 6,88$ & $0,009^{*}$ & $66,3 \pm 5,72$ & $65,4 \pm 5,75$ & 0,5 \\
\hline
\end{tabular}

Tabela 3: Comparação dos domínios e da qualidade de vida geral da pós-intervenção entre os grupos da massoterapia convencional e da drenagem linfática manual.

\begin{tabular}{cccc}
\hline Domínios & $M C(n=10)$ & $\operatorname{DLM}(n=10)$ & $p$ \\
\hline Físico & $63,5 \pm 6,48$ & $55,3 \pm 7,55$ & $0,01^{*}$ \\
Psicológico & $71,6 \pm 7,80$ & $67,4 \pm 9,16$ & 0,2 \\
Social & $80,8 \pm 18,0$ & $70,8 \pm 8,10$ & 0,1 \\
Meio Ambiente & $72,5 \pm 4,62$ & $68,1 \pm 7,49$ & 0,1 \\
QVG & $72,1 \pm 6,88$ & $65,4 \pm 5,75$ & $0,02^{*}$ \\
\hline
\end{tabular}

Dados apresentados em média e desvio padrão; MC: massoterapia convencional; DLM: drenagem linfática manual; n: número da amostra; QVG: qualidade de vida geral; p: nível de significância; *: diferença significativa $(p<0,05)$.

Portanto, conforme apresentado na figura 1, a técnica manual mais eficaz para a melhora da qualidade de vida das mães da comunidade de São Luiz Gonzaga foi à massoterapia convencional $(p=0,02)$.

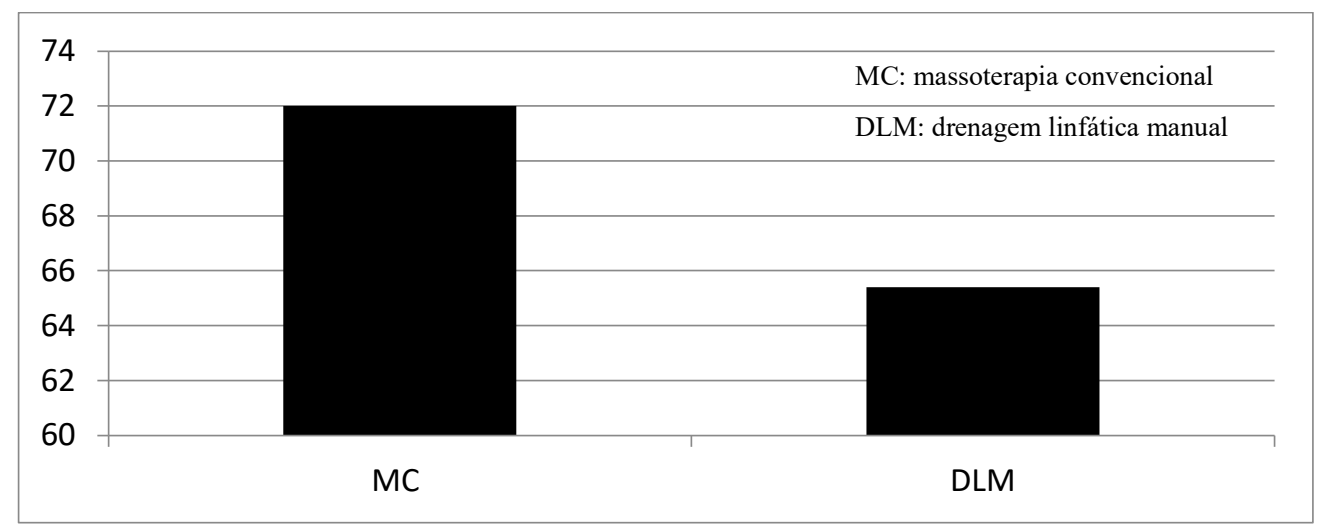

Figura 1: Comparação da qualidade de vida geral pós-intervenção entre os grupos da massoterapia convencional e da drenagem linfática manual.

\section{DISCUSSÃO}

O presente estudo demonstrou que existem diferenças significativas na melhora da qualidade de vida das mães submetidas às técnicas de MC e DLM, apesar disso, não foi encontrado na literatura estudos que relacionam as técnicas na melhora da QV, encontramos alguns que estudaram as técnicas separadamente.

Borges et al. (2013), realizaram uma pesquisa que objetivou comparar a qualidade de vida com o instrumento WHOQOL-100 e a melhora na amplitude de movimento, sendo verificada melhora significativa após o tratamento fisioterapêutico nos aspectos domínio físico, psicológico, nível de independência, relações sociais e meio ambiente e na amplitude articular dos movimentos. Assim, conclui que a intervenção fisioterapêutica composta de alongamentos, técnicas de relaxamento, massagens 
Comparação entre a drenagem linfática manual e a massoterapia convencional para a melhora da qualidade de vida das mães da comunidade de São Luiz Gonzaga

terapêuticas e eletroterapia foram benéficas para melhorar a qualidade de vida e a flexibilidade nos pacientes, vindo de encontro com o nosso estudo, no qual os domínios físico e meio ambiente apresentaram melhora significativa ( $p<0,05$, apresentado na tabela 2 ), além de que a intervenção foi eficaz para a melhora da qualidade de vida em geral.

Duran et al. (2016), realizaram um estudo em que aplicaram as técnicas de MC e, houve benefícios, diminuindo tensões, sintomas físicos, dores, e, consequentemente, uma melhora na qualidade de vida. Do mesmo modo, as mães do grupo da MC do presente estudo apresentaram uma melhora significativa na qualidade de vida em geral ( $p=0,009$, apresentado na tabela 2$)$. A MC é um conjunto de técnicas não invasivas que buscam manter a saúde e prevenir desequilíbrios, contribuindo com o bem-estar e melhora da qualidade de vida (NASCIMENTO et al., 2017).

De acordo com Coutinho et al. (2017) a DLM promove condições gerais de qualidade de vida, pois através do seu estudo no qual aplicou a DLM em seis mulheres, concluiu que a técnica possibilitou relaxamento, redução da ansiedade e auxiliou na qualidade do sono e bem-estar das participantes, mostrando-se satisfeitas quanto aos resultados percebidos na redução de edema e na melhora da qualidade de vida, corroborando com o presente estudo, no qual a técnica de DLM foi eficiente na melhora da qualidade de vida (tabela 3 ).

Ambas as técnicas ministradas foram significativas, porém, a técnica manual mais eficaz foi a MC (figura 1). Lin et al. (2001) confirmaram através do seu estudo que a MC proporciona relaxamento muscular e alívio da dor, e que ao toque produz respostas motoras, emocionais, comportamentais e de percepção corporal, concluindo que essa técnica visa à melhora da qualidade de vida, à readaptação e à reabilitação social e profissional e não apenas o alívio da dor.

\section{CONCLUSÕES}

Conclui-se que tanto a massoterapia convencional quanto a drenagem linfática manual promovem a melhora da qualidade de vida, apesar disso, no presente estudo a técnica manual mais eficaz para a melhora da qualidade de vida das mães estudadas, foi à massoterapia convencional.

\section{REFERÊNCIAS}

BORGES, M. C.. Avaliação da qualidade de vida e do tratamento fisioterapêutico em pacientes com cervicalgia crônica. Fisioter. Mov., v.26, n.4, 873-881, 2013.

CARREIRO, E. M.. Tratamento de rejuvenescimento facial pela estética e fisioterapia Dermato-Funcional: um pré-teste. Revista Científica da Escola da Saúde, v.1, n.2, 2012.

COSTA, E. C.; MEJIA, D. P. M.. Métodos terapêuticos dermato-funcionais no pós-operatório de abdominoplastia e lipoaspiração. Portal Biocursos, 2017.

COUTINHO, C. S.. Os efeitos da drenagem linfática manual do método leduc nos edemas dos membros inferiores das gestantes. Runa, 2017.

CREFITO 3. Conselho Regional de Fisioterapia e Terapia
Ocupacional de São Paulo. CREFITO 3, 2017.

DURAN, J. S.. Efeitos da massagem clássica na qualidade de vida em mulheres de 20 a 30 anos com síndrome da tensão pré-menstrual. Revista Científica do Unisalesiano, v.7, n.15, 2016.

FERREIRA, J. J.. Drenagem linfática manual nos sintomas da síndrome pré-menstrual: estudo piloto. Fisioterapia e Pesquisa, v.17, n.1, p.75-80, 2010.

HERNANDES, L. B. S.; MEJIA, D. P. M.. Bases legais para o exercício da fisioterapia Dermatofuncional e suas diversas aplicabilidades: o que o profissional precisa saber. Monografia (Especialização em Fisioterapia DermatoFuncional) - Faculdade Ávila, 2013.

LIN, T. Y.. Medicina física e reabilitação em doentes com dor 
crônica. Rev. Med., v.80, n.2, p.245-55, 2001.

MIURA, R. T.; PETEAN, E. B. L.. Paralisia cerebral grave: o impacto na qualidade de vida de mães cuidadoras.

Efdeportes, 2017.

NASCIMENTO, C.; FREITAS, M. S. Efeitos da massoterapia na qualidade do sono e desemprenho escolar: estudo de dois casos. Monografia (Bacharel em Fisioterapia) - Universidade São Francisco, 2017.

RADUNZ, V.; OLSON, J. Promoção de saúde e qualidade de vida entre mães de pré-adolescentes: um estudo etnográfico enfocado em Timbó/ SC- Brasil. Ver. Latino-Am.

Enfermagem, v.13, p.1135-41, 2005.
SALES, G. P.; FERREIRA, T. F.. Aplicação do questionário "WHOQOL-Bref" para avaliação da qualidade de vida nos participantes do projeto de promoção em saúde corra pela vida de São Roque Canaã/ES. Revista Brasileira de Prescrição e Fisiologia do Exercício, v.5, n.28, p.366-374, 2011.

SPINDOLA, T.; SANTOS, R. S.. Mulher e trabalho: a história de vida de mães trabalhadoras de enfermagem. Rev Latino-am Enfermagem, v.11, n.5, p.593-600, 2003.

A CBPC - Companhia Brasileira de Produção Científica (CNPJ: 11.221.422/0001-03) detém os direitos materiais desta publicação. Os direitos referem-se à publicação do trabalho em qualquer parte do mundo, incluindo os direitos às renovações, expansões e disseminações da contribuição, bem como outros direitos subsidiários. Todos os trabalhos publicados eletronicamente poderão posteriormente ser publicados em coletâneas impressas sob coordenação da Sustenere Publishing, da Companhia Brasileira de Produção Científica e seus parceiros autorizados. Os (as) autores (as) preservam os direitos autorais, mas não têm permissão para a publicação da contribuição em outro meio, impresso ou digital, em português ou em tradução. 